\title{
Maturation of Binocular Pattern Visual Evoked Potentials in Normal Full-Term and Preterm Infants from 1 to 6 Months of Age
}

\author{
MARIE-SYLVIE ROY, MAGDA BARSOUM-HOMSY, JACQUELINE ORQUIN, AND \\ JULIE BENOIT \\ Department of Ophthalmology, Hôpital Sainte-Justine [M.-S.R., M.B.-H., J.O., J.B.] and Department of \\ Ophthalmology, Université de Montréal [M.-S.R., M.B.-H., J.O.], Montreal, Quebec, Canada
}

\section{ABSTRACT}

\begin{abstract}
The purpose of this study was to evaluate the visual development of preterm infants from 1 to 6 mo of age, using the pattern visual evoked potentials (VEP) in response to three check sizes: 60,30 , and $15 \mathrm{~min}$ of arc. Pattern VEP were recorded in 24 full-term and 24 preterm infants (26-36 wk of gestation). The results showed a rapid visual maturation between 1 and 3 mo, followed by a slower progression over the next $3 \mathrm{mo}$, in both groups. The implicit time of the P100 wave of the pattern VEP was also found to shorten with increasing check sizes. The maturation of pattern VEP in preterm infants was shown to be related to their gestational (or corrected) age rather than their
\end{abstract}

postnatal age. The pattern VEP obtained in response to a 60 -min check size in preterm infants aged between 1.5 and $2.5 \mathrm{mo}$ (corrected age) showed a tendency for a faster maturation than those of full-term infants. Our results suggest that within the first 6 mo of age, pattern VEP response is useful to monitor visual development in full-term infants as well as in preterm infants using corrected age. (Pediatr Res 37: 140-144, 1995)

VEP, visual evoked potential

\section{Abbreviations}

ANOVA, analysis of variance
Preterm infants are known to be at risk for a number of vision-threatening conditions such as intraventricular hemorrhage (1), perinatal cerebral hypoxia (2), and retinopathy of prematurity (3), which can alter the normal maturation of the visual system. Studies (4-13) have been conducted to determine the time course of the normal visual development in preterm infants compared with full-term infants. Preterm infants are exposed to visual stimulation for an additional period of several weeks or months in comparison with infants born after the normal $40 \mathrm{wk}$ of gestation, and this precocious experience could have an effect on the developmental rate of visual functions. The results reported on the influence of visual experience versus the maturation of the visual system are often contradictory.

Earlier studies on preterm infants using behavioral methods (4-8) suggested that the extravisual experience would not influence the development of visual acuity, which appeared to be related to the infant's gestational (or corrected) age rather than its postnatal age. More recent reports using either the steady-state VEP testing (9) or preferential looking method $(10-11)$ revealed that the development of visual acuity in

Received October 11, 1993; accepted August 23, 1994.

Correspondence: Dr. Marie-Sylvie Roy, Department of Ophthalmology, Hôpital SainteJustine, 3175, Côte Ste. Catherine Road, Montreal, Quebec, Canada H3T 1C5.

Supported by a grant from FORMOEIL (Fondation pour la recherche sur les maladies de l'Oeil) and FRSQ 930214-103 from the Fonds de la recherche en santé du Québec. healthy preterm infants would be accelerated when compared with full-term infants. If there is an accelerating or facilitating effect in preterm infants resulting from an extrauterine experience before the expected term date, it needs to be taken into account to adjust the developmental norms of comparison between full-term and preterm infants and to help detect delayed or impaired visual development. Inasmuch as visual functions show a remarkably fast development during the first 6 mo of life in normal full-term infants (14-16), any effect of the extra visual experience in preterm infants would most likely find its expression in an acceleration of development at this earlier age.

The purpose of our study was to examine the maturation of pattern VEP in full-term compared with preterm infants from 1 to $6 \mathrm{mo}$ of age and to look for evidence of a stimulating or accelerating effect of the precocious visual experience in infants born prematurely. The pattern VEP method has been shown to produce finer acuity estimates than behavioral methods in young infants (17), and therefore this technique should be the most adequate testing method to index the maturation of the visual system in preterm infants. Finally, because the implicit time of the pattern VEP appears comparable to adult values by approximately 6 mo of age in full-term infants using large check sizes (18), its recording could lead to a more comprehensive spectrum of the visual maturation in full-term as well as preterm infants. 


\section{METHODS}

Subjects. Pattern VEP were recorded in 24 healthy preterm and 24 full-term infants, aged from 1 to 6 mo of corrected age at the time of testing. The mean age was 3.3 and 3.1 mo for the full-term and preterm infants (corrected age), respectively. Both groups were matched for sex: 15 females and nine males in each group. In the full-term group, the number of weeks of gestation at birth ranged from 38 to 42 wk (mean 39.9 wk). Premature infants were born after 26 to $36 \mathrm{wk}$ of gestation (mean $32 \mathrm{wk}$ ), and had no evidence of retinopathy of prematurity or neurologic or developmental abnormality for their gestational age at the time of testing. The birth weight ranged from 2404 to $4110 \mathrm{~g}$ (mean $3381 \pm 478 \mathrm{~g}$ ) in the full-term group and from 860 to $3240 \mathrm{~g}$ (mean $1713 \pm 622 \mathrm{~g}$ ) in the preterm group. All preterm infants underwent an ophthalmologic examination. Assessment of numbers of weeks of gestation at birth in preterm infants was based on mother's reports and pediatric examination. This study was approved by the human research committee of Hôpital Sainte-Justine; informed consent was obtained from a parent or legal guardian before the pattern VEP testing.

Recording procedures. The pattern VEP was recorded from a silver scalp active electrode positioned at $\mathrm{O}_{z}$, according to the International 10-20 system. The reference electrode was located on the right ear lobe $\left(A_{2}\right)$, and the left ear lobe $\left(A_{1}\right)$ served as ground. Electrode impedance was kept below $5 \mathrm{~K} \Omega$.

The stimulus display was a black and white checkerboard pattern generated by a visual pattern generator (Nicolet 1015, Nicolet Biomedical Instruments, Madison, WI) and presented on a large video-monitor screen of $58 \mathrm{~cm}$, subtending a visual field of $30 \times 30^{\circ}$. The check sizes subtended visual angles of 60,30 , or $15 \mathrm{~min}$ of arc. The checkerboard pattern was reversed at a rate of 1.3 alternations per second, producing a transient pattern VEP. The biopotentials were recorded with a Nicolet Pathfinder I signal averager (sweep time $500 \mathrm{~ms}$; prestimulus delay $50 \mathrm{~ms}$; recording bandwidth $1-100 \mathrm{~Hz}$ ), and an average of 50-100 responses were obtained for each check size. This range of sweeps was found to be adequate in recording P100 wave implicit time in young infants $(18,19)$. When additional sweeps are averaged, this prolongs the recording procedure and the infant is apt to lose interest. An artifact rejection was used to eliminate epochs contaminated by head and body movements. A hard copy of the pattern VEP responses in all subjects was printed with a Hewlett-Packard Desk Jet and the wave forms were then stored on floppy disk for further analysis.

Infants were seated on the parent's lap or held over the shoulder in front of the video-monitor screen at a distance of 70 $\mathrm{cm}$. They were tested binocularly with undilated pupils. To facilitate the testing, the room was maintained in a mesopic lighting without distractions. An observer stood next to the monitor and could easily judge the direction of the infant's gaze. The observer also made sounds with toys in front of the screen to attract the attention of the infant and to keep the infant's interest and alertness. The averaging process was interrupted with a manual switch if the reflection of the stimulus was not centered over the pupil. In all cases, we first recorded pattern VEP in response to 60 -min check size, and, when possible, 30- and 15-min check sizes were also tested.

Data analysis. After the onset of the stimulus, the first large positive peak appearing around $100 \mathrm{~ms}$ was identified as the P100 wave, and its implicit time calculated from the stimulus onset to the intercept of the two slopes of the wave. The identification of the P100 wave was done by two independent judges. When there was a discrepancy in the measure of the maximal peak time between the two judges, the mean of the two measures was taken. The differences in the implicit time of the P100 wave identified by the two judges did not exceed 6 ms. Implicit times were compared between full-term and preterm infants for all check sizes. Statistical significance was calculated with the use of a $t$ test or ANOVA. The best fit lines for the relationship between the implicit time of P100 as a function of age in full-term and preterm infants were determined by using the method of least squares of a logarithmic regression analysis and by calculating the coefficient of determination, $r^{2}$. Polynomial regressions were also calculated for the best fit of data points according to the method of least squares. The logarithmic regression was selected because it provides the highest coefficient of determination $\left(r^{2}\right)$ for the best visualization of the maturation curve of the P100 wave.

\section{RESULTS}

Young infants aged 1 to 6 mo old usually have short attention span and, as a result, not all infants have been successfully tested on the three check sizes. In the full-term group, the success rate of infants tested for the 60-, 30-, and 15 -min check size was $24 / 24,14 / 24$, and 10/24, respectively, whereas in the preterm infants it was $24 / 24,17 / 24$, and $10 / 24$, respectively. The difference in the success rate of testing between the two groups for each check size was not significant. The failure rate was mainly observed in the younger infants for the smallest check sizes.

Figure 1 shows the maturation of the P100 component of the pattern VEP for a 60-min check size obtained from six fullterm and six preterm infants (corrected age) aged between 1 and 6 mo. The results show a rapid maturation of the visual response as revealed by a large decrease in the implicit time of the P100 wave in both groups. The morphology of the pattern VEP in preterm infants is similar to that in full-term infants; the wave becomes more sharply defined as the infants get older, and there is a gradual development of an initial negative wave (N1) preceding the positive P100 wave.

Figure 2 shows the maturation of the P100 component in all full-term and preterm infants, using either postnatal (top graph) or corrected age (bottom graph), for the 60-min check size. For the full-term infants, we noted a rather fast maturation between 1 and $3 \mathrm{mo}$, followed by a slower progression for the next 3 mo. The P100 wave peaks at around $200 \mathrm{~ms}$ at $1 \mathrm{mo}$ of age, shifting to about $100-110 \mathrm{~ms}$ before $6 \mathrm{mo}$ of age. In preterm infants age-matched on the basis of postnatal age, a similar pattern is observed, but the implicit time of P100 consistently lagged behind those of the full-term infants. However, when the data are plotted using corrected age instead of postnatal age (bottom graph), the implicit time of P100 in the 

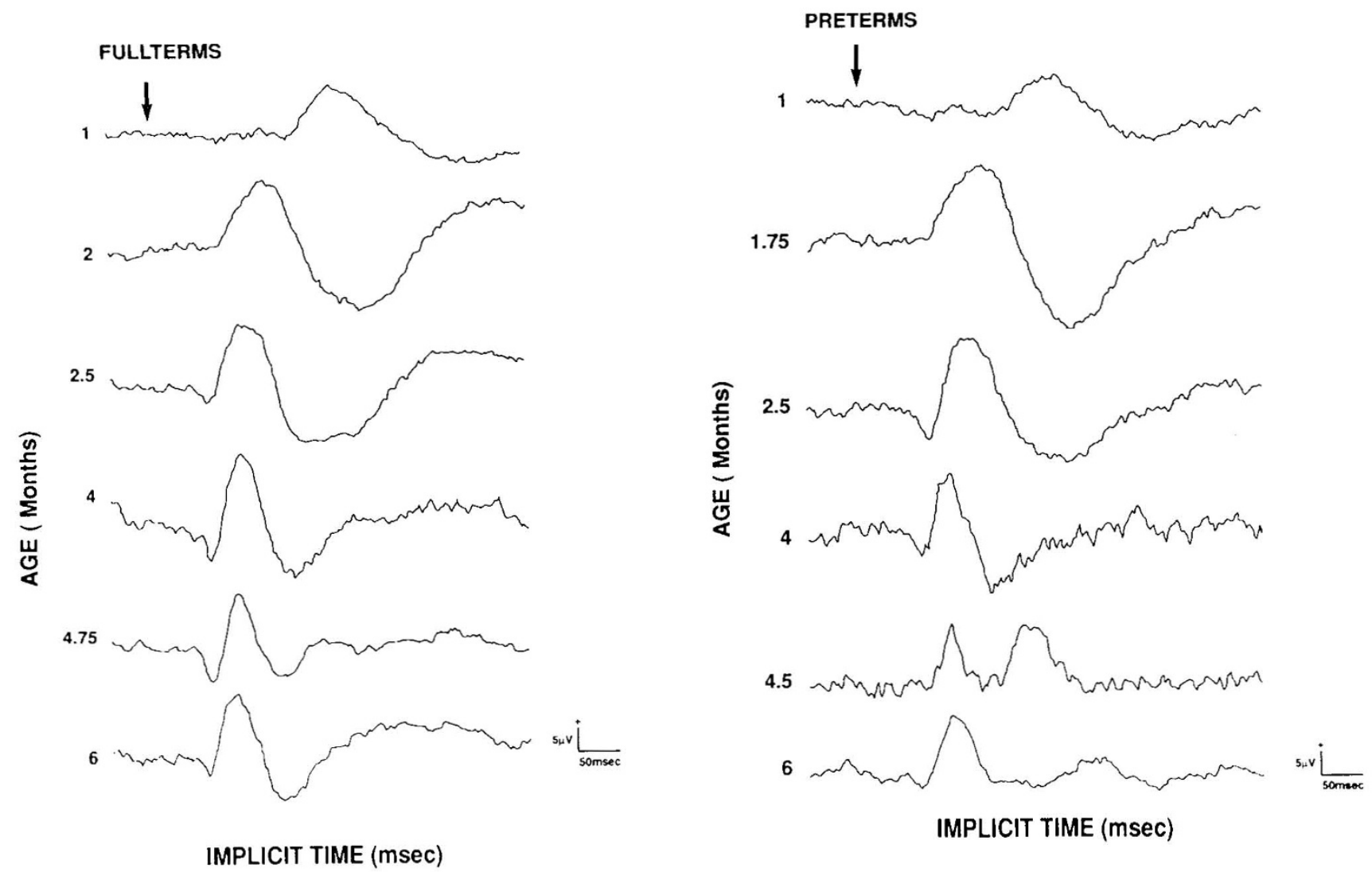

Figure 1. Pattern VEP obtained from six full-term infants (left) and six preterm infants of corrected age (right) between 1 and 6 mo. The potentials were evoked to a 60 -min check size.

preterm group is equal to, or slightly faster than, that in full-term infants, as suggested by the line of best fit. One notes that the best fit curves are not asymptotic as would be expected from our results for adult maturation of implicit time (which occurs normally at approximately 6 mo of age). However, the absence of asymptotic curves could be explained by the small number of infants in the 6- mo age group or the fact that we did not use a constant value for normal adult peak time. The equation of the best fit curve for the full-term group was $\mathrm{y}=$ $205.88-146.14 * \log (\mathrm{x}), r^{2}=0.750$, compared with $\mathrm{y}=$ $197.99-142.43 * \log (\mathrm{x}), r^{2}=0.839$, for preterm infants (corrected age), and $\mathrm{y}=257.35-178.17 * \log (\mathrm{x}), r^{2}=0.734$, for preterm infants (postnatal age). Interestingly, between the ages of 1.5 and 2.5 mo (corrected age), the implicit time of P100 in preterm infants appears to have a faster maturation compound than that in full-term infants (Fig. 2, inset), and this aspect seems related to a mild prematurity in five of the six preterm subjects. These five preterm infants were born between 33 to $35 \mathrm{wk}$ of gestation with a mean birth weight of $1947 \pm$ $765 \mathrm{~g}$. The mean implicit time for these preterm infants is $156.7 \pm 8.6 \mathrm{~ms}$ (mean age group $=1.74 \pm 0.37$ ) compared with $174.2 \pm 21.2 \mathrm{~ms}$ (mean age group $1.81 \pm 0.29, n=8$ ) for the full-term infants. This difference, however, is not statistically significant, nor is the difference between the two groups when infants of all ages are included (i.e. infants aged between 1 and $6 \mathrm{mo}$ ). We also did not find any relationship between the maturation of the pattern VEP implicit time in preterm infants as a function of birth weight.
Figure 3 shows the maturation of the P100 implicit time of VEP in all full-term and preterm (corrected age) infants as a function of the check sizes. For the full-term infants, the equations of the best fit curve for the 60-, 30-, and 15-min check size are as follows: $\mathrm{y}=208.66-152.06 * \log (\mathrm{x}), r^{2}=$ $0.812 ; \mathrm{y}=206.87-138.61 * \log (\mathrm{x}), r^{2}=0.796 ; \mathrm{y}=182.50$ $-85.244 * \log (\mathrm{x}), r^{2}=0,438$; for the preterm infants, they correspond to $\mathrm{y}=197.85-141.76 * \log (\mathrm{x}), r^{2}=0.839 ; \mathrm{y}=$ $183.95-101.87 * \log (\mathrm{x}), r^{2}=0.863 ; \mathrm{y}=207.05-113.42$ $* \log (\mathrm{x}), r^{2}=0.903$. For each group, an ANOVA was carried out with check sizes $(60,30$, and $15 \mathrm{~min}$ of arc) as factors. The implicit time of the P100 decreases significantly as a function of increasing check size in full-term infants $(F 2,18=676.974$; $p<0.0001)$ as well as in preterm infants $(F 2,20=397.974$; $p<0.0001)$. We also examined whether the influence of the check size on the maturation curve of the VEP was similar in preterm and full-term infants, and found no significant difference between the two groups ( $F 2,94=0.629 ; p=0.54$ ). There is also no significant difference in the implicit time of the VEP between preterm and full-term infants for each check size $(p>0.05)$.

Inasmuch as the maturation curve appears to be biphasic, with a faster maturation occurring within the first 3 mo of extrauterine life (reflected in the large decrements in implicit time observed from 1 to 3 mo of age), followed by a slower phase (more than 3 mo of age) (Figs. 2 and 3), we divided the infants into two subgroups (those 3 mo or younger and those older than $3 \mathrm{mo}$ ) to examine whether a difference between the 

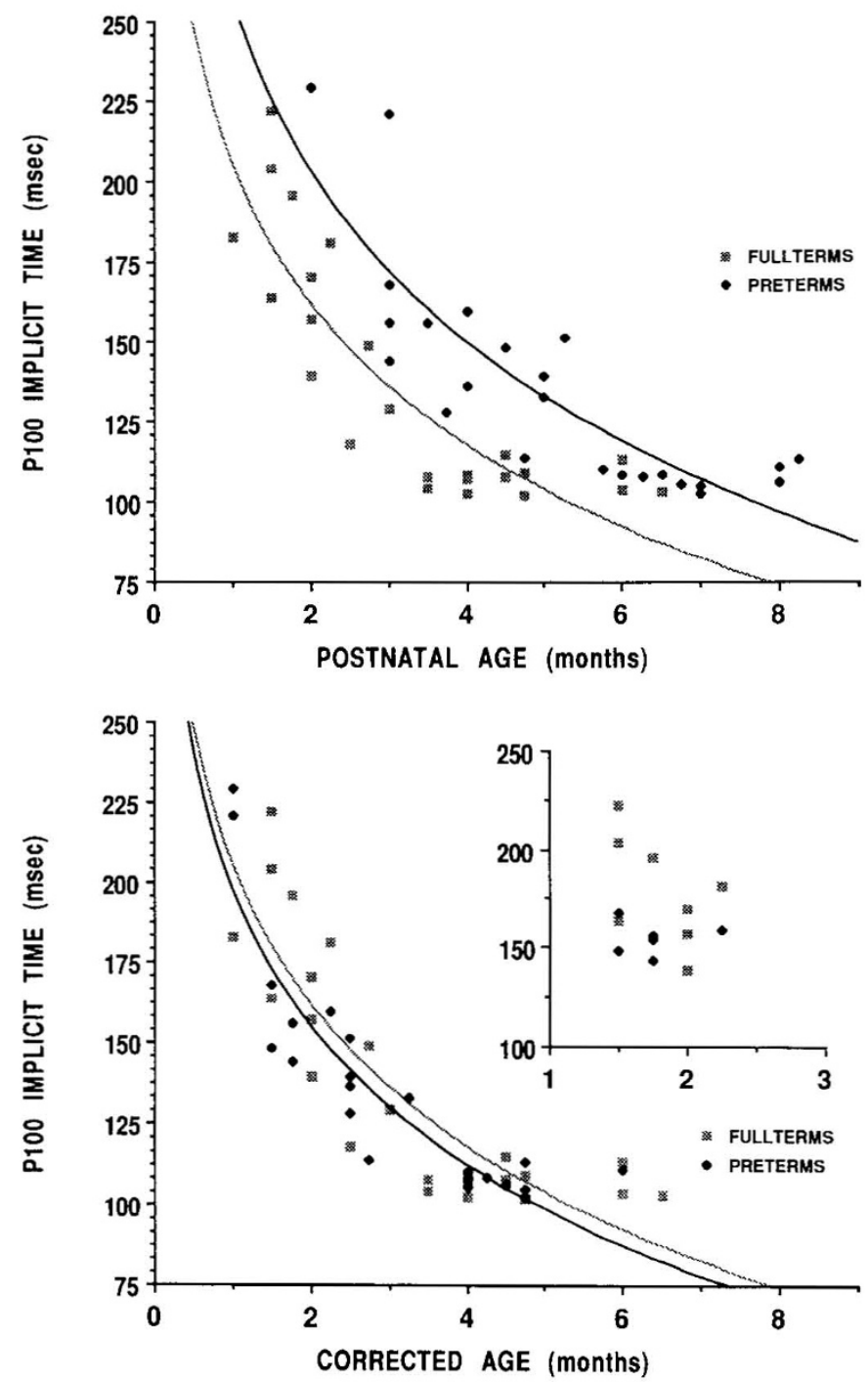

Figure 2. Implicit time of the $\mathrm{P} 100$ wave obtained from a 60 -min check size as a function of age for preterm $(n=24)$ and full-term infants $(n=24)$. Top graph, The age of the preterm infants is expressed in postnatal age (i.e. number of weeks after birth). Bottom graph, Age of preterm infants corrected to its equivalent gestational age. Inset (bottom graph), Enlargement of the graph for the data obtained between 1.5 and $2.5 \mathrm{mo}$. The equations of the best fit curve for full-term infants (gray lines) and preterm infants (dark lines) are reported in the text.

preterm and full-term infants could be detected during one of these two phases of maturation. For each subgroup of age, an ANOVA was carried out on the subjects (full-term and preterm infants) with check sizes (60, 30, and 15 min of arc) as factors. (For the younger subgroup, only the 30 - and 60 -min check sizes were included, because the success rate of VEP testing with a 15-min check size was too small for quantitative analysis: $n=3$ in preterm infants and $n=1$ in full-term infants.) No significant difference was found between full-term and preterm infants on the maturation of the VEP as a function of check sizes, for both the younger $(F 1,35=0.094 ; p=0.76)$ and older subgroups $(F 2,50=1.011 ; p=0.37)$ of age. There is also no significant difference between the preterm and full-term infants for each check size, in both the younger and older subgroups $(p>0.05)$.
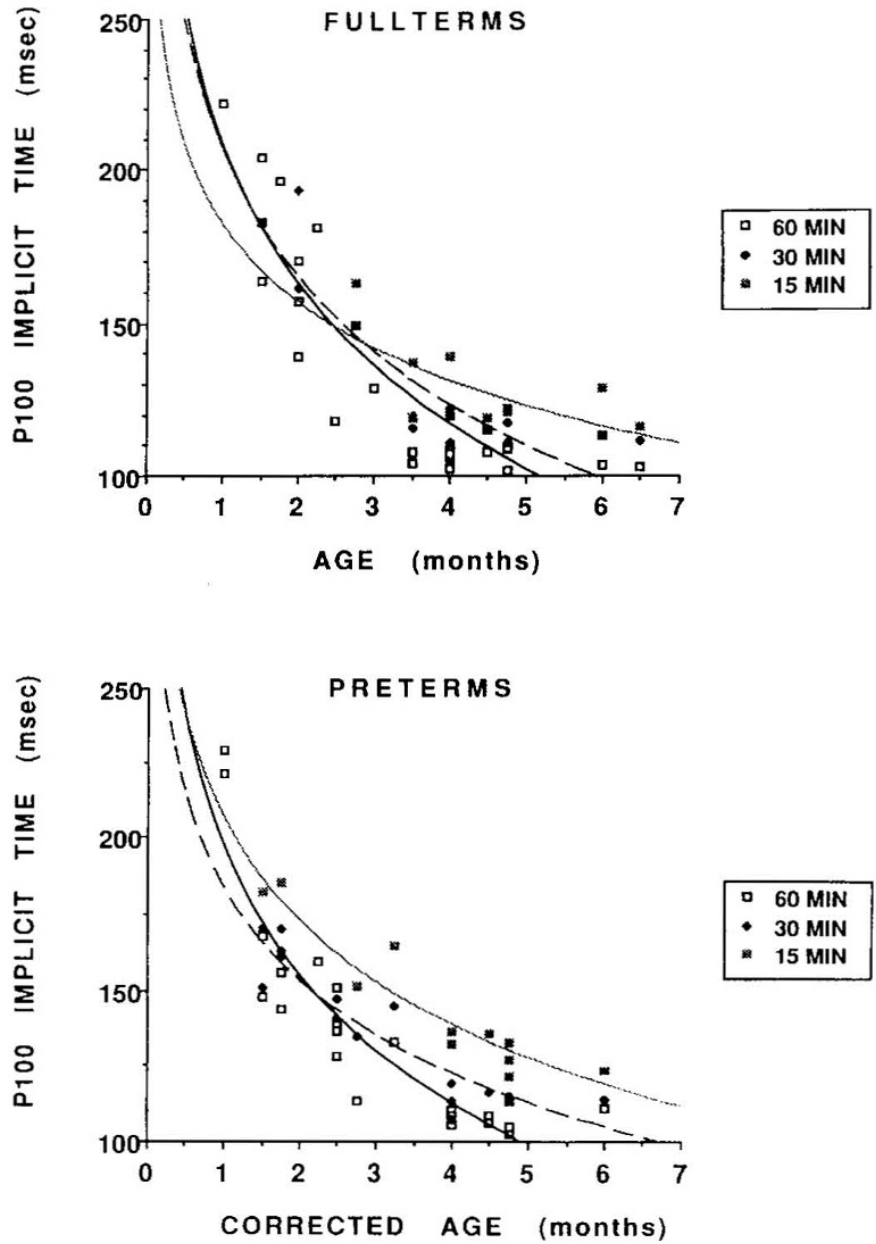

Figure 3. Implicit time of the P100 wave obtained from 60-, 30-, and 15-min check size as a function of age for the full-term (top graph) and preterm (bottom graph) groups. The equations of the best fit curve for 60- (dark lines), 30- (broken lines), and 15-(gray lines) min check sizes are reported in the text.

\section{DISCUSSION}

The aim of this study was to examine visual maturation in healthy preterm infants using pattern VEP and to look for evidence of a positive or facilitating effect of the precocious visual experience in infants born before the expected term date. Our results showed a rapid maturation of the implicit time of the pattern VEP between 1 and $3 \mathrm{mo}$, followed by a slower progression of the maturation over the next $3 \mathrm{mo}$, in full-term infants as well as in preterm infants (corrected age). When comparing the P100 obtained with a 60 -min check size in full-term and preterm infants using either postnatal or corrected age, our results appear correlated to gestational rather than postnatal age (Fig. 2). These results are in agreement with earlier studies on preterm infants, indicating that acuity development is related to gestational age (4-8).

The influence of the various check sizes on the maturation of the implicit time of the pattern VEP was also examined in both groups. We noted a shortening of the implicit time with an increase in check size in both groups (Fig. 3). The latter was also reported in full-term infants $(18-20)$ and on a small group of preterm infants (20). Moreover, during the 6-mo period examined, the influence of the check size on the implicit time 
of the VEP did not differ between full-term and preterm infants, nor was a difference found between preterm and fullterm infants for each check size. The same finding was also noted when considering the rapid and the progressive phases of maturation. (However, we were not able to include the 15 -min check size for the younger subgroup because of the lower success rate of testing.) Hence, the maturation of the VEP in preterm infants seems to follow a developmental pattern comparable to that of the full-term infants. These results suggest that the early visual stimulation experienced by the preterm infants would not contribute significantly or permanently to the maturation of the pattern VEP implicit time, at least as monitored with the check sizes used in this study. Therefore, corrected age appears to be a better indicator of binocular acuity (i.e. maturation) in preterm infants aged from 1 to $6 \mathrm{mo}$ using the pattern VEP. The regression curves (at least for the 60 -min check sizes, where all the subjects were included), could be used as guiding lines to follow visual maturation in full-term and specially in preterm infants because of their higher incidence of visual problems (2-3).

However, there are some aspects of our results suggesting, in agreement with more recent studies $(10-13)$, that visual development in preterm infants could be accelerated when compared with full-term infants of the same gestational age. We noted that between the ages of 1.5 and 2.5 mo (corrected age), the implicit time of the P100 in some preterm infants seems to have a faster maturation compared with full-term infants for the 60-min check size (Fig. 2, inset). However, this difference did not reach a level of significance. Sokol and Jones (20) also noted a shorter implicit time of the P100 in preterm infants aged between 2 and 3 mo (corrected age) with the use of large checks of 120 and $240 \mathrm{~min}$ of arc, but not for small checks (7.5 and $15 \mathrm{~min}$ of arc). Inasmuch as it has been suggested that the rate of myelinization seems to be facilitated by several factors, including light exposure (21), these observations need further clarification.

In conclusion, the use of pattern VEP seems useful to assess the ongoing maturation of the visual system in full-term infants as well as in healthy preterm infants as young as 1 to 6 mo of age. Moreover, it is appropriate for general clinical assessment of the maturation of visual function with the pattern VEP to use adjusted age to correct for prematurity.
Acknowledgments. The authors thank Dr. Anne-Claude Bonnin, Department of Paediatrics, Dr. Harry Bard, Department of Neonatalogy, and all the staff for their collaboration. We also thank all the parents who agreed to have their infants participate in this study.

\section{REFERENCES}

1. Clarke CE, Clyman RI, Roth RR, Sniderman SH, Lane B, Ballard RB 1981 Risk factor analysis of intraventricular hemorrhage in low-birth-weight infants. J Pediatr 99:625-628

2. Groenendaal F, van Hof-van Duin J, Baerts W, Fetter WPF 1989 Effects of perinatal hypoxia on visual development during the first year of (corrected) age. Early Hum Dev 20:267-279

3. Phelps DL 1981 Vision loss due to retinopathy of prematurity. Lancet 1:606-610

4. Fantz RL, Fagan JF, Miranda SB 1975 Early visual selectivity. In: Cohen LB, Salapatek P (eds) Infant Perception: From Sensation to Cognition. Academic Press, New York, pp 249-345

5. Dobson V, Mayer DL, Lee CP 1980 Visual acuity screening of preterm infants. Invest Ophthalmol Vis Sci 19:1498-1505

6. Morante A, Dubowitz LMS, Levene M, Dubowitz V 1982 The development of visual function in normal and neurologically abnormal preterm and preterm infants. Dev Med Child Neurol 24:771-784

7. Van Hof-van Duin J, Mohn G 1984 Vision in the preterm infant. In: Prechtl WFR (ed) Continuity of Neural Functions from Pre- to Postnatal Life. Blackwell, Philadelphia, pp 93-115

8. Van Hof-van Duin J, Mohn G, Fetter WPF, Mettau JW, Baerts W 1983 Preferential looking acuity in preterm infants. Behav Brain Res 10:47-50

9. Norcia AM, Tyler CW, Piecuch R, Blyman R, Grobstein J 1987 Visual acuity development in normal and abnormal preterm human infants. J Pediatr Ophthalmol Strabismus 24:70-74

10. Van Hof-van Duin J, Mohn G 1986 The development of visual acuity in normal fullterm and preterm infants. Vision Res 26:909-916

11. Searle CM, Horne SM, Bourne KM 1989 Visual acuity development: a study of preterm and full-term infants. Aust $\mathrm{N} \mathrm{Z} \mathrm{J}$ Ophthalmol 17:23-26

12. Roy MS, Lachapelle P, Leporé F 1987 Maturation of optokinetic nystagmus and the role of visual experience in normal and preterm infants. Invest Ophthalmol Vis Sci 28(suppl):313(abstr)

13. Roy MS, Lachapelle P, Leporé F 1989 Maturation of the optokinetic nystagmus as a function of the speed of stimulation in fullterm and preterm infants. Clin Vis Sci 4:357-366

14. Spekreijse H 1983 Comparison of acuity tests and pattern evoked potential criteria: two mechanisms underlie acuity maturation in man. Behav Brain Res 10:107-117

15. Braddick OJ, Atkinson J 1983 Some recent findings on the development of human binocularity: a review. Behav Brain Res 10:141-150

16. Fiorentini A, Pirchio M, Spinelli D 1983 Development of retinal and cortical responses to pattern reversal in infants: a selective review. Behav Brain Res 10:99106

17. Dobson V, Teller DY 1978 Visual acuity in human infants: a review and comparison of behavioural and electrophysiological studies. Vision Res 18:1469-1483

18. McCulloch DL, Skarf B 1991 Development of the human visual system: monocular and binocular pattern VEP latency. Invest Ophthalmol Vis Sci 32:2372-2381

19. Grose J, Harding GFA, Wilton AY, Bissenden JG 1989 The maturation of the pattern reversal VEP and flash ERG in preterm infants. Clin Vis Sci 4:239-246

20. Sokol S, Jones K 1979 Implicit time of pattern evoked potentials in infants: an index of maturation of spatial vision. Vision Res 19:747-755

21. Hoyt CS, Nickel BL, Billson FA 1982 Ophthalmological examination of the infants. Surv Ophthalmol 26:177-190 